\title{
Respostas Cardiovasculares Agudas ao Exercício de Força Realizado em Três Diferentes Formas de Execução
}

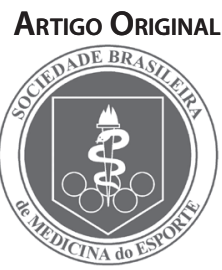

\author{
Acute Cardiovascular Responses to Strength Exercise in Three Different Execution Patterns
}

\author{
Walace David Monteiro',2 \\ Denis Alves de Souza ${ }^{1}$ \\ Maurício Nunes Rodrigues ${ }^{1,3}$ \\ Paulo de Tarso Veras Farinatti $1^{1,2}$ \\ 1. Laboratório de Atividade Física e \\ Promoção da Saúde. Universidade \\ do Estado do Rio de Janeiro \\ (LABSAU-UERJ) \\ 2. Programa de Pós-Graduação \\ em Ciências da Atividade Física \\ - Universidade \\ Salgado de Oliveira (UNIVERSO) \\ 3. Instituto de Desenvolvimento \\ e Pesquisa em Atividades Físicas \\ (IDEATIVA)
}

Endereço para correspondência: Laboratório de Atividade Física e Promoção da Saúde - Universidade do Estado do Rio de Janeiro (LABSAU-UERJ).

Rua São Francisco Xavier, 524, $8^{\circ}$ andar, Sala 8133, Bloco F.

Maracanã, Rio de Janeiro, RJ, CEP: 20559-900

Email:wdm@uerj.br

Submetido em 17/07/2007

Aceito em 06/09/2007

\begin{abstract}
RESUMO
A forma de condução do movimento no exercício de força pode afetar as respostas cardiovasculares agudas. $O$ estudo comparou as respostas agudas de freqüência cardíaca (FC), pressão arterial sistólica (PAS) e duplo produto (DP) durante o exercício extensão de joelhos nas formas unilateral, bilateral e alternada. A amostra foi composta por 10 voluntários com idades entre 23 e 39 anos (28 \pm 5 anos). Inicialmente os sujeitos realizaram testes de 10 repetições máximas (RM) nas execuções bilateral e unilateral. Em seguida, foram medidas as respostas cardiovasculares nas três formas de execução. Em todos os casos, foram feitas três séries de 10 RM, com 2 min de intervalo entre elas. As medidas para cada forma de execução foram feitas com $24 \mathrm{~h}$ de intervalo. Os valores das variáveis observadas, considerando cada série para as formas bilateral, unilateral e alternada foram, respectivamente: $F C 1=108,6$ vs 117,3 vs 110,$9 ; F C 2=120,0$ vs 130,1 vs 130,$0 ; F C=125,3$ vs 137,1 vs 135,$2 ; \mathrm{PAS} 1=143,8$ vs 150,0 vs 144,$8 ;$ PAS2 $=155,8$ vs 159,6 vs 158,$8 ;$ PAS3 $=159,6$ vs 166,0 vs 164,$8 ;$ DP $1=15609,8$ vs 17873,4 vs 16246,6; DP3=20016,8 vs 23175,2 vs 22572,0. Não foram encontradas diferenças significativas nas respostas de FC, PAS e DP para as distintas formas de execução em cada série isoladamente. A partir da $2^{\text {a }}$ série os valores absolutos para as respostas de FC e DP foram maiores nas formas unilateral e alternada, em comparação com a execução bilateral $(p<0,05)$. Conclui-se que a forma de execução não exerceu influência nas respostas cardiovasculares durante o exercício. Contudo, respostas maiores foram sistematicamente verificadas para as execuções unilateral e alternada, principalmente em séries múltiplas. Isso pode ser relevante para a prescrição do exercício de força em populações com problemas cardiovasculares.
\end{abstract}

Palavras-chave: freqüência cardíaca, pressão arterial, duplo produto, treinamento, aptidão física.

\begin{abstract}
The pattern of execution can influence the acute cardiovascular responses during exercise. The study observed the heart rate (HR), systolic blood pressure (SBP), and double product (DP) during leg extension executed in three different ways - single-leg (SL), double-leg (DL) and alternate (AL). Ten healthy volunteers (28 \pm 5 years-old) performed three bouts of 10 maximum repetitions of each execution pattern, with a two min interval between sets. The sessions for $\mathrm{SL}, \mathrm{DL}$, and $\mathrm{AL}$ were held at different days, with a $24 \mathrm{~h}$ interval. The results for the observed variables in each execution pattern and bout were respectively: HR $1=108.6$ vs 117.3 vs 110.9; $\mathrm{HR} 2=120.0$ vs 130.1 vs $130.0 ; \mathrm{HR}=125.3$ vs 137.1 vs $135.2 ; \mathrm{SBP} 1=143.8$ vs 150.0 vs $144.8 ; \mathrm{SBP} 2=155.8$ vs 159.6 vs $158.8 ;$ SBP3 $=159.6$ vs 166.0 vs $164.8 ; \mathrm{DP} 1=15609.8$ vs 17873.4 vs $16246.6 ; \mathrm{DP} 3=20016.8$ vs 23175.2 vs 22572.0. There were no significant differences between the execution patterns in each bout. After the 2 nd bout, the absolute HR and DP responses were higher for $\mathrm{AL}$ and $\mathrm{SL}$, in comparison with $\mathrm{DL}$. We conclude that the execution pattern did not significantly influence the acute cardiovascular responses. However, higher responses were systematically observed for the $A L$ and $S L$ patterns in a multiple series design. This information should be taken into account in programs aimed at subjects with cardiovascular disease.
\end{abstract}

Keywords: heart rate, blood pressure, double product, strength training, physical fitness.

\section{INTRODUÇÃO}

É praticamente consensual que um maior conhecimento sobre as respostas cardiovasculares ao treinamento de força em diferentes situações é relevante para a segurança do praticante, principalmente quando as suas condições clínicas implicam maiores riscos. Nesse sentido, não é surpreendente que diversos estudos tenham investigado as respostas cardiovasculares ao treinamento de força em situações diversas ${ }^{(1-6)}$. A forma de condução dos exercícios tem papel importante no planejamento das sessões de treinamento, influenciando na magnitude da tensão exercida pelos músculos e, portanto, nas respostas esperadas em longo prazo. Por exemplo, realizar o exercício de forma uni ou bilateral modifica a quantidade de massa muscular acionada, afetando as cargas suportadas e o tempo de execução do exercício. Uma vez influenciando na intensidade do trabalho muscular, as formas de condução dos exercícios também podem modificar substancialmente as respostas cardiovasculares a eles associadas.

É possível encontrar na literatura estudos que se debruçaram sobre 
a influência da forma de execução sobre as respostas cardiovasculares agudas ${ }^{(1,7-10)}$. Em todos eles foram executadas repetições submáximas ou até a exaustão, tomando-se como base cargas obtidas em testes de 1 repetição máxima (RM). Polito et al.(11) criticaram estudos dessa natureza, enfatizando que o número de repetições alcançado na execução bilateral pode não ser igual ao da execução unilateral (no caso de repetições até a fadiga). Mais ainda, que o estímulo pode ser insuficiente para maximizar as respostas cardiovasculares e possibilitar a identificação de diferenças entre as formas de execução (no caso de repetições submáximas). Esses autores investigaram as respostas de freqüência cardíaca (FC), pressão arterial (PA) e duplo produto (DP) nas execuções uni e bilaterais na extensão do joelho, conduzidas até a exaustão, valendo-se de cargas obtidas em testes de 12 RM, buscando reproduzir situações habituais de treinamento.

O estudo de Polito et al. ${ }^{(11)}$ trouxe contribuições importantes, observando duas das mais freqüentes formas de execução utilizadas no contexto da prescrição do treinamento da força. Contudo, outra forma de execução, comumente utilizada e não investigada, envolve a alternância dos seguimentos requisitados para a realização do exercício. A exemplo das formas de execução uni e bilateral, a execução alternada pode associar-se a um padrão bastante específico de respostas cardiovasculares agudas. No entanto, uma revisão de literatura nas principais bases de dados (Medline, Sports Discus, Scielo e Lilacs) não conseguiu localizar estudos que tivessem testado essa hipótese.

Desse modo, pesquisas que comparem as respostas ao treinamento de força nas três diferentes formas de execução podem oferecer informações importantes no que se refere à segurança da prática do exercício. Assim, o objetivo do presente estudo foi comparar as respostas agudas de freqüência cardíaca, pressão arterial sistólica e duplo-produto nas execuções bilateral, unilateral e alternada no exercício extensão de joelhos, realizado em diferentes séries com cargas correspondentes a 10RM.

\section{MATERIAIS E MÉTODOS}

\section{Amostra}

A amostra foi composta por dez voluntários do sexo masculino com idades entre 23 e 39 anos (idade $=28 \pm 5$ anos; massa $=76 \pm 8$ kg; estatura $=1,78 \pm 0,1 \mathrm{~m}$ ). Para a seleção da amostra respeitaram-se os seguintes critérios: a) prática regular do treinamento de força por pelo menos seis meses consecutivos; b) questionário Par-Q negativo. Como critérios de exclusão consideraram-se os seguintes aspectos: a) uso de substâncias que pudessem alterar as respostas cardiovasculares nas situações de repouso e esforço; b) realização de exercícios nas 24 horas que antecederam os testes; c) problemas ósteo-mio-articulares que pudessem interferir na realização do exercício proposto. Todos os voluntários assinaram um termo de consentimento pós-informado, conforme sugerido pela Resolução 196/96 do Conselho Nacional de Saúde para experimentos com seres humanos. O estudo foi aprovado por comitê de ética institucional.

\section{PROCEDIMENTOS}

\section{Coleta de dados}

A coleta de dados foi realizada em seis dias. O primeiro foi destinado à aplicação do questionário PAR-Q e anamnese, para seleção dos sujeitos e detecção de algum critério de exclusão. No segundo e terceiro dias, foram realizados testes de 10RM. A opção por dois dias de testes teve por finalidade determinar a reprodutibilidade das cargas. No quarto, quinto e sexto dias foram coletados os dados referentes às respostas cardiovasculares agudas nas distintas formas de execução do exercício. As medidas foram feitas sempre nos mesmos horários, entre 8 e 10 da manhã, cada dia sendo destinado à observação das respostas cardiovasculares agudas em uma dada forma de execução. As sessões de exercício foram separadas por um intervalo de 24 horas. A primeira forma de execução foi feira pela técnica do quadrado latino, para que cada um iniciasse por uma execução diferente.

\section{Formas de Execução do Exercício e Número de Séries Reali- zadas}

O exercício de extensão do joelho foi executado nas formas unilateral, bilateral e alternada. No primeiro caso, realizaram-se todas as repetições com uma das pernas, para depois fazê-las com a outra. $\mathrm{Na}$ forma bilateral, as repetições foram feitas por ambos os membros simultaneamente. Como a própria denominação sugere, a execução alternada foi feita por alternância das pernas para cada repetição do movimento.

Em todas elas, foram realizadas três séries com cargas fixas inicialmente para que fosse executadas 10 RM, com intervalo de dois minutos entre as séries. Para que fossem determinadas as cargas, foi realizado um teste de 10RM nas formas de execução bilateral e unilateral. A carga utilizada para a execução alternada foi igual àquela adotada na execução unilateral do joelho, devido à similaridade das execuções que usam a mesma massa muscular e angulação de movimento, sendo desnecessário realizarem testes de carga para ambas as execuções. A diferença entre as formas de execução residiu apenas na alternância de cada perna para a condução do exercício.

As angulações dos movimentos foram iguais, já que o equipamento utilizado permitia esse tipo de ajuste. Dessa forma, regulou-se o equipamento para que os movimentos não excedessem $90^{\circ}$ de flexão do joelho. Quanto ao movimento de extensão, esse foi conduzido até a angulação máxima do movimento. Cabe notar que o padrão respiratório foi controlado, recomendando-se que se expirasse durante a fase concêntrica do movimento.

\section{Teste de 10RM}

Os testes de carga foram realizados em dois dias não consecutivos. A cada dia testou-se apenas uma forma de execução, determinada por ordem alternada.

Para cada forma de execução foi testada a reprodutibilidade de carga para 10RM, de modo que a variação entre as duas seqüências de exercícios não ultrapassasse os 5\%. Foram, além disso, testadas a diferença e a associação entre os resultados das seqüências, respectivamente pelo teste t-Student e $r$ de Pearson $(p<0,05)$. Foi dado um intervalo de 10 minutos entre cada teste de $10 \mathrm{RM}$. $\mathrm{O}$ equipamento utilizado foi a cadeira extensora marca Technogym ${ }^{\varpi}$, modelo Rehabilition Device (Milão, Itália).

Visando reduzir a margem de erro nos testes de 10RM, foram adotadas as seguintes estratégias ${ }^{(12)}$ : a) instruções padronizadas foram fornecidas antes do teste, de modo que o avaliado estivesse ciente de toda a rotina que envolvia a coleta de dados; b) o avaliado foi instruído sobre a técnica de execução do exercício durante as diferentes etapas de sua realização; c) o avaliador estava atento quanto à posição adotada pelo praticante no momento da medida, pois pequenas variações no posicionamento das articulações envolvidas no movimento poderiam acionar outros músculos, levando a interpretações errôneas dos escores obtidos; d) foram definidas as seguintes etapas de execução dos exercícios: posição inicial e desenvolvimento, esta última compreendendo as fases concêntrica e excêntrica da contração. Esse procedimento foi adotado para facilitar a contagem dos movimentos realizados de forma correta; e) estímulos verbais foram realizados a fim de manter alto o nível de estimulação durante a execução do exercício; f) os pesos 
adicionais utilizados no estudo foram previamente aferidos em balança de precisão; g) o intervalo entre as tentativas para obtenção da maior carga para 10RM foi fixado em cinco minutos.

\section{Medida das Variáveis Cardiovasculares}

A aferição das variáveis cardiovasculares foi realizada nas condições de repouso e exercício, com auxílio dos mesmos equipamentos. A descrição das medidas é apresentada a seguir:

a) Repouso - Inicialmente, os indivíduos permaneceram sentados por 10 minutos. A FC foi medida continuamente, sendo registrado o menor valor apresentado nesse período, com uso de um freqüencímetro Polar ${ }^{\oplus}$ modelo A5 (Kempele, Finlândia). A medida da PA foi realizada pelo método auscultatório, utilizando-se um esfigmomanômetro Tycos ${ }^{\oplus}$ modelo 7050 (Miami, EUA) e um estetoscópio Rappaport ${ }^{\circledR}$ modelo V416 (Vernon Hills, USA). Para a aferição, cada indivíduo posicionou o braço esquerdo, relaxado, em superfície plana à altura do ombro. A fixação do manguito no braço ocorreu com aproximadamente $2,5 \mathrm{~cm}$ de distância entre sua extremidade inferior e a fossa antecubital. Após inflar o manguito, iniciou-se o processo de esvaziamento até distinguirem-se o $1^{\circ}$ e o $5^{\circ}$ ruídos de Korotkoff, correspondentes aos valores sistólico e diastólico, respectivamente. Tal procedimento foi adotado com base nas recomendações da American Heart Association ${ }^{(13)}$.

b) Exercício - A FC foi medida continuamente, sendo registrado o maior valor apresentado durante as repetições do exercício. Para medida da PA, levou-se em conta que as respostas de pico provavelmente ocorrem durante as últimas repetições de uma série. Por isso, os dados foram aferidos entre a antepenúltima e a última repetição de cada série ${ }^{(14,15)}$. Desse modo, o início do esvaziamento do manguito coincidiu com o início da antepenúltima repetição. Isso possibilitou tempo suficiente para a determinação da PAS ao final da última repetição. A medida da PA foi realizada por avaliador experiente, cuja reprodutibilidade da medida nas situações de repouso e esforço foi determinada previamente. Durante a coleta de dados, os voluntários foram orientados a não realizar manobra de Valsalva, bem como movimentar ou contrair o braço esquerdo. Os procedimentos de medida da PA durante exercícios resistidos com uso do método auscultatório foram recentemente validados, com excelente associação entre seus resultados e valores obtidos com técnica fotopletismográfica ${ }^{(15)}$.

\section{Tratamento Estatístico}

A reprodutibilidade do avaliador na medida da pressão arterial nas situações de repouso e exercício, foi testada pelo coeficiente de correlação intra-classe. Para comparar as respostas de FC, PAS e DP nas diferentes séries e formas de execução do exercício investigado, utilizou-se uma ANOVA de duas entradas para medidas repetidas, seguidas da verificação post-hoc de Scheffé. Em todos os casos, o nível de significância adotado foi de $p<0,05$. Todos os cálculos foram feitos com auxílio do software Statistica $6.0^{\circ}$ (Tulsa, EUA).

\section{RESULTADOS}

A reprodutibilidade do teste de 10 RM foi considerada satisfatória. Considerando as seqüências com variação inferior a 5\%, obteve-se uma correlação de $0,88(p<0,05)$ entre elas, não tendo sido identificadas diferenças significativas $(t=1,2 ; p>0,05)$. Os resultados do coeficiente de correlação intra-classe para a reprodutibilidade das medidas da PAS e PAD foram de 1,00 na situação de repouso e 0,94 (PAS) e 0,91 (PAD) ao final de 10RM. A Figura 1 ilustra os resultados referentes à FC nas distintas formas de condução do exercício. Foram encontradas diferenças significativas entre o repouso e todas as formas de execução. Para cada série considerada isoladamente, não foram verificadas diferenças nas respostas de FC entre as diferentes formas de execução.
Contudo, levando-se em conta o efeito somativo do número de séries, verificou-se uma tendência de valores menores de FC na execução bilateral. Diferenças significativas foram verificadas da primeira para a terceira série nas execuções unilateral e alternada, esta última também apresentando diferença da primeira para a segunda série.

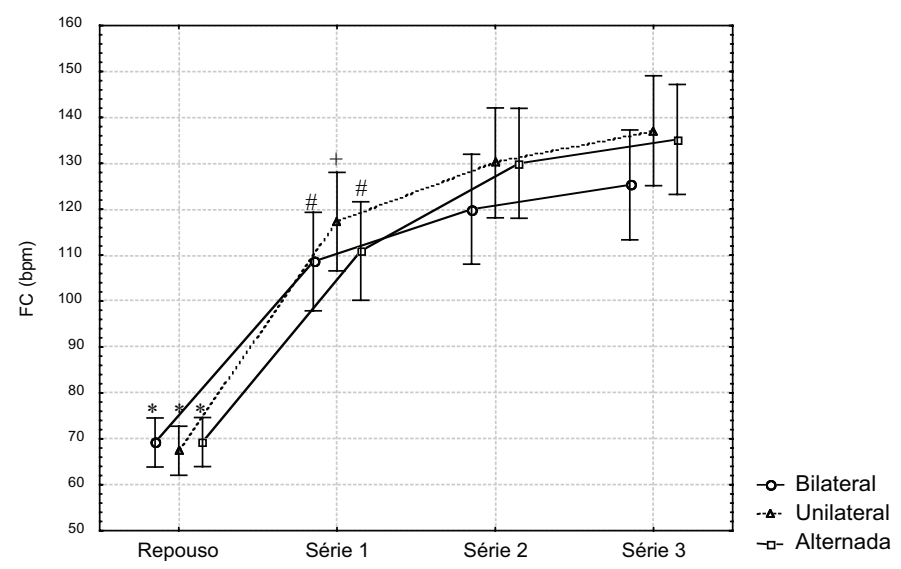

Figura 1. Respostas da freqüência cardíaca (FC) nas diferentes formas de execução do exercício cadeira extensora. ${ }^{*}$ Diferença significativa do repouso para todas as séries $(p<0,05)$; \# Diferença significativa entre a $1^{\mathrm{a}}$ e $3^{\mathrm{a}}$ séries, nas execuções unilateral $(p=0,03)$ e alternada $(p=0,03) ;+$ Diferença significativa da $1^{\text {a }}$ para a $2^{2}$ série na execução alternada $(p=0,02)$. As barras representam intervalos de confiança a $95 \%$.

A Figura 2 apresenta os resultados obtidos para a PAS. Assim como na $F C$, houve diferenças significativas entre o repouso e todas as formas de execução. Não foram encontradas diferenças de pressão entre as formas de execução, ao compararem-se as séries isoladamente ou considerando seu efeito somativo. Contudo, valores sistematicamente mais altos de PAS foram verificados nas formas de execução unilateral e alternada, enquanto menores valores associaram-se à execução bilateral.

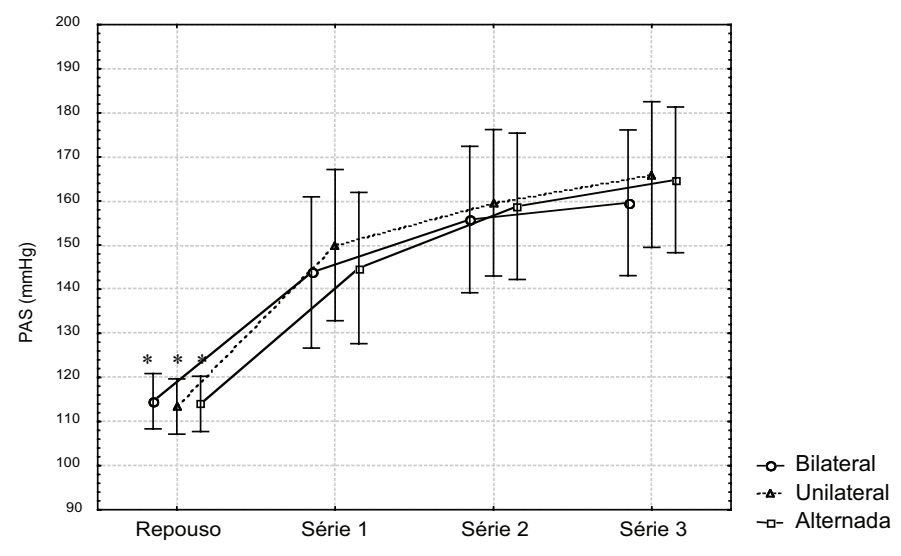

Figura 2. Respostas da pressão arterial sistólica (PAS) nas diferentes formas de execução do exercício cadeira extensora. * Diferença significativa do repouso para todas as séries $(p<0,05)$. As barras representam intervalos de confiança a $95 \%$.

A Figura 3 ilustra os resultados referentes ao DP. Como esperado, foram verificadas diferenças significativas do repouso para o esforço em todas as formas de execução. Para cada série isolada, não foram verificadas diferenças nas respostas de DP nas três formas de execução. Contudo, a análise do efeito cumulativo do número de séries revelou valores sistematicamente menores de DP para a execução bilateral. Diferenças significativas foram verificadas entre a primeira e a terceira série nas execuções unilateral e alternada. 


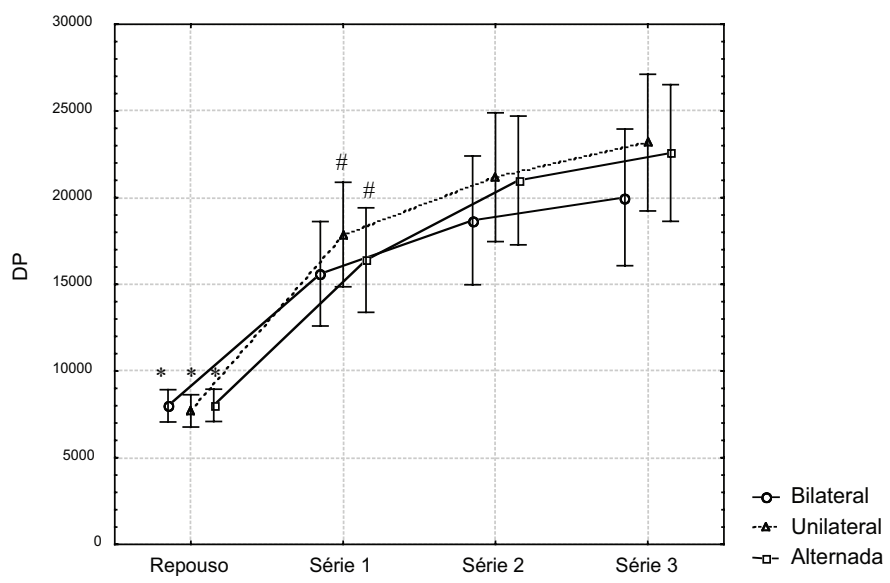

Figura 3. Respostas do duplo produto (DP) nas diferentes formas de execução do exercício cadeira extensora. * Diferença significativa do repouso para todas as séries $(p<0,05)$; \# Diferença significativa entre a $1^{\text {a }}$ e $3^{\text {a }}$ séries, nas formas de execução unilateral $(p=0,02)$ e alternada $(p=0,04)$. As barras representam intervalos de confiança a $95 \%$.

\section{DISCUSSÃO}

Antes de iniciar a discussão dos resultados, convém tecer um comentário sobre uma possível limitação do estudo, referente à utilização do método auscultatório para a medida da PA. É claro que os resultados absolutos de PA fornecidos por técnicas invasivas, como o cateterismo intra-arterial, são mais confiáveis e válidos. No entanto, por ser invasivo, o cateterismo reveste-se de riscos consideráveis, como dor, espasmo arterial, trombose, estenose, síncope vaso-vagal e hemorragia, entre ou$\operatorname{tros}^{(10)}$. Para alguns, a aplicação desse tipo de procedimento em estudos como este extrapolaria os limites éticos da investigação científica ${ }^{(13)}$.

É importante reconhecer que a medida da PA pelo método auscultatório tende a subestimar os valores absolutos da PA durante os exercícios, principalmente naqueles de característica submáxima. Contudo, em atividades mais intensas, comparando-se os valores obtidos pelo método auscultatório e outros, como o cateterismo intra-arterial ou fotopletismografia, a relação entre eles tende a ser mantida ${ }^{(11,15-17)}$. Ou seja, considerando que a tendência de subestimação ocorre de forma sistemática, a associação de uma atividade com maiores ou menores valores de PA pode ser detectada ${ }^{(15)}$.

Para todas as formas de execução do exercício, as variáveis investigadas apresentaram valores significativamente maiores quando comparados com aqueles obtidos em situação de repouso. Isso já era esperado, visto que, durante o exercício dinâmico observa-se um aumento da FC, da PAS e, por conseqüência, do DP(18,19). No que diz respeito à influência da forma de execução dos exercícios nas respostas cardiovasculares agudas ao exercício resistido, a literatura ainda é escassa. Recentemente, Polito et al. ${ }^{(11)}$ investigaram as respostas de FC, PA e DP na extensão do joelho realizada de forma bilateral e unilateral. Em conclusão, os autores verificaram que a forma de execução não influenciaria as respostas cardiovasculares agudas ao exercício. Apesar disso, os autores chamam atenção para o fato de que as respostas de FC, PAS e DP, durante a execução bilateral, tenderam a se elevar em relação à execução unilateral.

Nesse sentido, a literatura tem demonstrado que haveria um efeito somativo do número de séries sobre as respostas agudas de FC, PAS e DP. Gotshall et al. ${ }^{(20)}$ analisaram os efeitos de séries consecutivas sobre a resposta da PA, após a execução de três séries de 10 repetições no exercício leg-press. Foi observado que, quanto maior o número de séries em determinado exercício, maiores as respostas obtidas para a PA. Os resultados do presente estudo parecem alinhar-se com essa concepção.

Na comparação entre as séries para uma mesma forma de execução, as respostas de FC apresentaram elevação estatisticamente significativa para as execuções alternada e unilateral. Isso pode estar relacionado ao maior tempo para execução das repetições nessas formas de execução, em comparação à execução bilateral. Como destacado por Falkel et al. ${ }^{(22)}$, o comportamento da FC durante uma série isolada do exercício de força associa-se eminentemente ao tempo de duração do estímulo. Apesar da maior carga absoluta mobilizada, o fato de o exercício ser feito pelas duas pernas simultaneamente resultaria em um menor tempo total de execução das repetições. Logo, as formas alternada e unilateral associar-se-iam a um período maior de oclusão arterial sem repouso, com provável impacto sobre os níveis de $F C^{(14)}$.

Já a PAS pareceu não sofrer influência do número de séries. Todavia, deve-se notar que, ainda que as diferenças não tenham sido significativas, as respostas de pressão revelaram-se sistematicamente mais elevadas para execução unilateral. Essa forma de execução envolve menor massa muscular e, conseqüentemente, menor carga de trabalho absoluta. Nesse contexto, apesar disso não ter sido uma preocupação central do estudo, cabe dizer que o tempo de tensão associado à execução unilateral foi o maior entre as três formas investigadas. Mesmo que estudos futuros sejam necessários para confirmar essa hipótese, pode-se pensar que isso afete as respostas de pressão arterial durante o exercício, em virtude de um aumento da intensidade da carga e conseqüente oclusão vascular decorrente da tensão muscular aplicada (7,23).

O DP teve comportamento similar à FC, diferenciando-se estatisticamente da primeira para a terceira séries nas execuções unilateral e alternada. Isso era esperado, uma vez que modificações na FC e PAS refletem-se nos valores do $D P^{(14)}$. Ainda que não se tenham revelado diferenças estatísticas entre as formas de execução, é importante destacar que, na execução bilateral, houve uma certa tendência de os valores de FC, PAS e DP serem menores que os observados nas demais formas de execução. Em comparação com as formas alternada e unilateral, os valores obtidos para a forma bilateral foram, para FC, PAS e DP, inferiores em cerca de 10 bpm, 5 mmHg e 2500 bpm•mmHg, respectivamente. Isso ocorreu, principalmente, a partir da segunda série, o que deveria ser levado em consideração em estudos futuros e, talvez, no planejamento dos programas de treinamento para indivíduos com maior risco cardiovascular.

O intervalo entre as séries de um exercício é um fator que também pode influenciar nas respostas cardiovasculares agudas ao esforço. Farinatti et al. (21) investigaram a influência de diferentes intervalos entre as séries nas respostas cardiovasculares agudas a séries múltiplas do exercício de força. Apesar de as respostas cardiovasculares terem sido mais exacerbadas nas séries com menores tempos de recuperação, verificou-se igualmente um efeito somativo do número de séries nas respostas ao esforço. Uma maneira de se administrarem intervalos entre séries é trabalhar de forma alternada. De certo modo, o espaçamento entre as execuções foi testado no presente estudo, já que além das execuções unilateral e bilateral, foi investigado o efeito da execução alternada nas respostas cardiovasculares. Com isso, apesar de os intervalos entre as séries terem sido fixos para todas as formas de execução, na forma alternada haveria maior tempo de repouso dentro de uma mesma seqüência, ou seja, repouso entre as repetições. Ainda assim, não houve diferenças significativas entre a forma alternada e as outras, o que leva a pensar que esse tipo de estratégia não teria repercussão importante sobre as respostas de PAS e FC.

Haslam et al. ${ }^{(7)}$ sugerem que a quantidade de carga mobilizada no 
treinamento exerce influência decisiva sobre as respostas cardiovasculares no esforço. Os resultados dos estudos de Polito et al. ${ }^{(11)}$, bem como os aqui encontrados, parecem não confirmar essa possibilidade. Enquanto no primeiro executaram-se séries com cargas relativas a 12RM, no presente estudo as séries tinham cargas correspondentes à 10RM. Isso leva a pensar que existiria um patamar mínimo de carga que, conjugado a um dado número de repetições, seria capaz de repercutir de forma mais significativa nas respostas cardiovasculares ao esforço. As cargas manipuladas em 10 e 12 repetições, talvez por serem muito próximas, não foram capazes de exercer efeito diferente nas variáveis estudadas. Outros estudos são necessários para melhor entender os efeitos cardiovasculares das relações entre carga e repetições, nas diferentes formas de execução do treinamento.

Na tentativa de controlar a velocidade de movimento, Polito et al.(11) fixaram o tempo para a execução das fases concêntrica e excêntrica do movimento. Partiu-se da premissa de que a fadiga muscular poderia levar a um aumento do tempo de tensão, com menor velocidade total de movimento ${ }^{(24)}$. Desse modo, ao estabelecerem-se tempos fixos para execução das fases concêntrica e excêntrica, podem-se obter respostas cardiovasculares diferentes daquelas realmente observadas em uma sessão habitual de treinamento de força. A comparação entre os resultados deste estudo e os veiculados por Polito et al. ${ }^{(11)}$ não logrou confirmar essa possibilidade.

Como dito, no experimento supracitado ${ }^{(11)}$ cada série foi realizada em 48 segundos, tendo sido controlados os tempos de contração com auxílio de metrônomo (dois segundos para cada fase de contração). No nosso entender, a marcação dos tempos para execução das fases concêntrica e excêntrica do exercício descaracteriza o treinamento comumente realizado. Nossa experiência com o treinamento da força faz-nos pensar que haveria um aumento do tempo de tensão na fase concêntrica e uma redução na fase excêntrica, o que se agravaria na medida em que as séries acumulam-se. Como os praticantes vão alcançando maiores níveis de fadiga com a evolução das repetições, existe maior dificuldade em manter o tempo de execução do exercício na fase concêntrica, sendo esse tempo aumentado.

Em contrapartida, na fase excêntrica da contração, o movimento pode ser mais facilmente controlado pelo executante, existindo uma tendência em manter ou mesmo reduzir o tempo total de execução do movimento. De fato, isso foi ratificado por nossos resultados e, na prática, pode ser facilmente constatado em treinamentos que se valem de séries múltiplas de exercícios resistidos. No presente estudo o tempo para a execução das repetições não foi previamente determinado, a fim de aproximar o experimento das situações reais de treinamento. Com isso, o tempo médio de execução na forma de condução bilateral revelou-se o menor entre as três formas investigadas.

\section{CONCLUSÃO}

Em conclusão, não houve diferenças significativas entre as respostas cardiovasculares agudas associadas às formas de execução bilateral, unilateral e alternada em 10 RM do exercício extensão de joelhos. No entanto, a realização do exercício de forma bilateral acarretou, sistematicamente, menores valores de FC, PAS e DP, enquanto os maiores valores foram identificados sempre para a execução unilateral e alternada. Estudos futuros são necessários para um melhor entendimento da influência daquelas formas de execução sobre as variáveis analisadas. Outrossim, é preciso determinar a relevância das respostas presentemente obtidas em programas de treinamento delineados para indivíduos cuja condição clínica inspire, durante a prática do exercício físico, maiores cuidados em termos cardiovasculares.

Todos os autores declararam não haver qualquer potencial conflito de interesses referente a este artigo.

\section{REFERÊNCIAS BIBLIOGRÁFICAS}

1. McDougall JD, Tuxen D, Sale DG, Moroz JR, Sutton JR. Arterial blood pressure response to heavy resistance exercise. J Appl Physiol 1985; 58: 785-90.

2. Hardy DO, Tucker $L A$. The effects of a single bout of strength training on ambulatory blood pressure levels in 24 mildly hypertensive men. Am J Health Promot 1999; 13: 69-72.

3. Bermon S, Rama D, Dolsi C. Cardiovascular tolerance of healthy elderly subjects to weight-lifting exercises. Med Sci Sports Exerc 2000; 32: 1845-8.

4. Roltsch MH, Mendez T, Wilund KR, Hagberg JM. Acute resistive exercise does not affect ambulatory blood pressure in young men and women. Med Sci Sports Exerc 2001; 33: 881-6.

5. Polito MD, Simão R, Senna GW, Farinatti PTV. Efeito hipotensivo do exercício de força realizado em intensidades diferentes e mesmo volume de trabalho. Rev Brás Med Esporte 2003; 9: 69-73.

6. Simão R, Fleck SJ, Polito MD, Monteiro, WD, Farinatti PTV. Effects of resistance training intensity, volume, and session format on the postexercise hypotensive response. J Strength Cond Res 2005; 19: 853-8.

7. Haslam DRS, McCartney N, Mckelvie RS, McDougall JD. Direct measurements of arterial blood pressure during formal weightlifting in cardiac patients. J Cardiopulm Rehabil 1988; 8: 213-25.

8. Wiecek EM, McCartney N, Mckelvie RS. Comparison of direct and indirect measures of systemic arterial pressure during coronary artery disease. Am J Cardiol 1990; 66: 1065-9.

9. Fleck SJ, Dean LS. Resistance-training experience and pressor response during resistance exercise. J Appl Physiol 1987; 63: 116-20.

10. Leite TC, Farinatti PTV. Estudo da freqüência cardíaca, pressão arterial e duplo-produto em exercícios resistidos diversos para grupamentos musculares semelhantes. Rev Bras Fisiol Exerc 2003; 2: 68-88.

11. Polito MD, Rosa CC, Schardong P. Respostas cardiovasculares agudas na extensão do joelho realizada em diferentes formas de execução. Rev Bras Med Esporte 2004; 10: 173-6.

12. Monteiro W, Simão R, Farinatti P. Manipulação na ordem dos exercícios e sua influência sobre o número de repetições e percepção subjetiva do esforço em mulheres treinadas. Rev Bras Med Esporte 2005; 11: 146-50.
13. Perloff D, Grim C, Flack J, Frohlich E, Hill M, McDonald M. Human blood pressure determination sphygmomanometry. Circulation 1993; 88: 2400-7.

14. Farinatti PTV, Assis BFC. Estudo de freqüência cardíaca, pressão arterial e duplo-produto em exercícios contra-resistência e aeróbio contínuo. Rev Bras Ativ Fís Saúde 2000; 5: 5-16.

15. Polito MD, Farinatti PTV, Lira VA, Nóbrega ACL. Blood pressure assessment during resistance exercise: comparison between auscultation and Finapres. Blood Press Monit 2007; 12: 81-6.

16. Raftery EB. Direct versus indirect measurement of blood pressure. J Hipertens 1991; S10-2.

17. Jürimäe T, Jürimäe J, Pihl E. Circulatory response to single circuit weight and walking training session of similar energy coast in midle-age overweight females. Clin Physiol 2000; 20: 143-9.

18. American College of Sports Medicine. ACSM's Guidelines for exercise testing and prescription. $7^{\text {th }}$ ed. Baltimore, Lippincott Williams \& Wilkins: 2006.

19. Pollock ML, Franklin GJ, Balady GJ, Chaitman BL, Fleg JL, Fletcher B et al. AHA Science Advisory. Resistence exercise in individuals with and without cardiovascular disease. Benefits, rationale, safety, and prescription. An advisoty from the Committee on Exercise, Rehabilitation, and Prevention, Council on Clinical Cardiology, American Heart Association. Circulation 2000; 101: 828-33.

20. Gotshall R, Gootman J, Byrnes W, Fleck S, Valovich T. Noninvasive characterization of the blood pressure response to the double-leg press exercise. JEP-on line 1999; 2: 1-6.

21. Farinatti PTV, Simão R, Nóbrega ACL, Polito MD. Fracionamento das séries em exercício de força pode associar-se a maior estresse cardiovascular que séries contínuas. In: XXVI Simpósio Internacional de Ciências do Esporte 2003; 11:91.

22. Falkel JE, Fleck SJ, Murray TF. Comparison of central hemodynamics between powerlifters and body builders during resistance exercise. J Appl Sport Sci Res 1992; 6: 24-35.

23. Sale DG, Moroz DE, McKelvie RS, MacDougall JD, McCartney N. Comparison of blood pressure response to isokinetic and weight-lifting exercise. Eur J Appl Physiol Occup Physiol 1993; 67: 115-20.

24. Fleck SJ, Kraemer WJ. Design resistence training programs. $3^{\text {rd }}$ ed. Champaign: Human Kinetics, 2004. 